\title{
Zur Politik medialer Dispositive
}

\author{
SAMUEL SIEBER
}

Politik, so scheint es, beginnt und endet medial. In Zeiten der „Mediokratie“ (Meyer 2001), des „Politainments“ (Dörner 2005) oder gar der „Postdemokratie“ (Crouch 2008) ist jede ,Politik“ von Medien kolonisiert. Mit dem Fernsehen in medialer Vorreiterrolle haben die ,klassischen“ Massenmedien längst die Öffentlichkeit anästhesiert, politische Parteien marginalisiert und jede repräsentative Demokratie degradiert (vgl. Meyer 2001: 149ff). Andernorts werden Medien dagegen als Initianten politischer Revolutionen und Grundlage moderner Demokratiesierungsprozesse gefeiert: Computer, Mobiltelefon und Internet erlauben oppositionelle Organisation, partizipative Krisenkommunikation und die simultane Information einer Weltöffentlichkeit $^{1}$. Der augenscheinlichen Paradoxie zum Trotz wird das Beziehungsgeflecht zwischen Medien und Politik vorzugsweise mit teleologischen Denkfiguren von Mittel und Zweck und mittels bipolarer Machtkonzepte theoretisiert. Kommunikations- wie politikwissenschaftlich überwiegen Dependenz- und Instrumentalisierungstheorien: ,Politik' wird entweder als abhängig von den Medien betrachtet, oder Medien gelten als für politische Zwecke instrumentalisiert.

Im Gegensatz zu solchen Modellen sind Medien darauf hin zu befragen, welche Politiken sie selbst betreiben und inwiefern sie überhaupt als ,politisch ' interpretierbar sind. In ihren dispositiven Strukturen betrachtet, offenbaren sich Medien als politische Ordnungen von Sag- und Sichtbarkeiten: es

1 Schon 1978 schreibt Michel Foucault: „Die iranische Revolte breitet sich mittels Tonbandkassetten aus.“ (Foucault 2003), gleichenorts war 2009 von einer „Twitter-Revolution“ die Rede. Die Demonstrationen in Tunesien oder Ägypten im Frühjahr 2011 schienen ebenfalls von den ,Neuen Medien“ Twitter, Facebook oder Youtube begünstigt (vgl. von Rohr 2011). Zu einer ausführlicheren Auseinandersetzung mit „Medien in der Krise“ vgl Sieber 2011 
ist die Ordnungsmacht einer medialen ,Polizei ${ }^{{ }^{2}}$, die im Feld des Politischen Aussagen und Sichtbarkeiten hervorbringt und regelt. Gleichzeitig sind Interventionen in diese Ordnungen, wie sie in medialen Störungen und Transformationen auszumachen sind, als Momente politischer ,Teilhabe' und Aushandlung interpretierbar. Bevor Medien eine, vorgängige' Politik besetzen, bevor sie von Politik oder ,Polizei` instrumentalisiert werden, sind sie also selbst politisch wirkungsreich. Die so oft bipolar gedachte Machtkonstellation zwischen Medien und Politik verschiebt sich damit: kein „,massives und homogenes Phänomen der Herrschaft“ (Foucault 1978a: 82) prägt das medien-politische Beziehungsfeld. Vielmehr funktioniert Macht als „,netzförmige Organisation“, ,als etwas, das zirkuliert oder vielmehr als etwas, das nur in Art einer Kette funktioniert" (ebd.). Zu beleuchten sind deshalb jene ,Maschen der Macht", die Medien und Politik in produktive Wechselwirkung bringen: strategisch geordnete Sag- und Sichtbarkeiten in medialen Mikro- und Makrodispositiven, d.h. in Dispositiven der Einzelmedien und medial geprägten, einem ganzen gesellschaftlichen Feld immanenten Makrodispositiven. Die Politik dieser medialen Dispositive ist die ihrer ,politischen Differenz" (Marchart 2010): zwischen institutionalisierten Formen der Politik (franz. la politique), der Ordnungsmacht einer ,Polizei` (franz. la police) und der Substanz und Eigenschaft eines Politischen (franz. le politi$q u e)$, das jeder Politik vorgängig ist.

\section{Mediale Mikro- Und MaKrodispositive}

In der deutschsprachigen Film- und Medienwissenschaft war und ist der Dispositivbegriff stark durch die ,metapsychologische[n] Betrachtungen des Realitätseindrucks“ Jean-Louis Baudrys (1999) geprägt. Michel Foucaults Konzept des Dispositivs fiel dagegen lange Zeit hinter diese „kinotheoretische Verbreitung“ des Begriffs eines „kinobegeisterten Zahnarztes“ zurück (Hans 2001: 22). Dabei sucht Foucaults Diskurs- und Machtanalytik gerade nach den Ordnungen der Sag- und Sichtbarkeit, um ihre Macht- und Subjekteffekte aufzudecken (vgl. Deleuze 1991: 156f).

Nach Foucault verfügen Dispositive sowohl über eine charakteristische Struktur wie auch über spezifische Funktionen: erstens handelt es sich bei einem Dispositiv um ein ,entschieden heterogenes Ensemble, das Diskurse, Institutionen, architekturale Einrichtungen, reglementierende Entscheidungen, Gesetze, administrative Massnahmen [...], umfasst“ (Foucault 1978b:

2 ,Polizei“ ist dabei im Foucault'schen Sinne zu verstehen: als Verwaltungs- und Ordnungsmacht, d.h. als Kalkül und Technik, „,die die Schaffung einer flexiblen, aber dennoch stabilen und kontrollierbaren Beziehung zwischen der inneren Ordnung des Staates und dem Wachstum seiner Kräfte ermöglicht“ (Foucault 2004: 451). 
119), wobei das Dispositiv als das Netz zwischen diesen Elementen zu verstehen ist. Die Diskurse der Rechtsprechung und das Gefängnis oder die Diskursivierung des Sexes und der klinische Blick formen in Foucaults Analysen die Dispositive der Strafjustiz und der Sexualität (vgl. Foucault 1977; 1987); im Fokus steht immer die Verbindung diskursiver und nichtdiskursiver Elemente. Zweitens antwortet ein Dispositiv als Formation immer auf einen „Notstand“, d.h. auf eine krisenhafte Dringlichkeit, weshalb es eine vorwiegend strategische Funktion hat (Foucault 1978b: 120f). Foucaults Dispositive bilden sich durch strategische Imperative wie Hungersnöte (vgl. Foucault 2004: 62f) oder ein „Millieu der Delinquenz“ (Foucault 1978b: 122) aus. Diese Strategien diskursiver Ordnungen zeigen sich exemplarisch in einer Theorie der Interdiskursivität, wie sie unter anderen Jürgen Link entwickelt hat: Dispositive ordnen Spezialdiskurse (d.h. funktional ausdifferenzierte Diskurse wie z.B. die Wissenschaft) und Elementardiskurse (z.B. Diskurse der Alltagsrede oder des Alltagswissens) zu Interdiskursen (als Beziehungen und Transformationen zwischen Spezial- und Elementardiskursen) (vgl. Link 2006: 19). Interdiskurse, wie sie beispielsweise in der massenmedialen Vermittlung wissenschaftlicher Forschung auszumachen wären, sind das Resultat der strategischen Prävalenz eines Dispositivs, womit sich umgekehrt Interdiskursivität, ,als spezifische Operativität von ,Dispositiven“ zuordnen“ lässt (Link/Link-Heer 1990: 93).

Dispositive sind auch in ihrer Größe und Ausbreitung von Heterogenität geprägt. Bei Foucault bestehen sie einerseits ,,aus einer diffusen, heterogenen Mannigfaltigkeit, aus Mikro-Dispositiven“ und verweisen andererseits ,auf ein Diagramm, auf eine Art abstrakte Maschine, die dem ganzen gesellschaftlichen Feld immanent ist" (Deleuze 1996: 15). Zu unterscheiden sind also Mikrodispositive und größere, komplexere Makrodispositive. In medienwissenschaftlicher Anwendung spricht Matthias Thiele von einzelmedialen „Medien-Dispositiven“, die zugleich als „,konstitutive Teilelemente" größerer Makrodispositive fungieren können (Thiele 2009: 44f). Beispielsweise sind die von Foucault aufgedeckten Dispositive der Sexualität oder der Strafjustiz nicht a priori ,medial', wohl aber von bestimmten medialen und kommunikativen Praktiken wie wissenschaftlichen Taxonomien oder einem Geständniszwang geprägt.

Der Vorteil einer Applikation des Dispositivbegriffs auf Einzelmedien liegt nun darin, ,dass die verschiedenen Bereiche eines Mediums - wie Technik bzw. Apparatur, institutioneller Kontext, ökonomische Dimension, Raum- und Produktionspraktiken, ästhetische Verfahren und Stile, Wahrnehmungs-, Gebrauchs- und Rezeptionsweisen - zusammengedacht werden können“ (Thiele 2009: 41). Gleichzeitig sind Medien als Konglomerate mehrerer (medialer) Mikrodispositive vorstellbar, wobei immer medienspezifische und intermediale Eigenschaften hervortreten (vgl. Thiele 2009: 45). Das Dispositiv des modernen, mit Kamera, Internet, synchronisierbarem Kalender und E-Mail-Client ausgerüsteten Mobiltelefons kann beispielsweise als Konglomerat aus den Dispositiven des Telefons, des Internets, des 
Videos und Fernsehens, der Fotografie und des Büros gelten ${ }^{3}$. Als Politik medialer wie medial geprägter Dispositive sind in diesem Sinne neben der strategischen Prävalenz der Interdiskursivität auch Strategien der Intermedialität zu vermuten: politische und mediale Diskurse verbinden sich in und zwischen den Dispositiven der Medien und der Politik zugleich interdiskursiv und intermedial - und ziehen spezifische Machtverhältnisse und Subjekteffekte nach sich.

\section{Mediale Ordnungen der Sag- Und Sichtbarkeit}

Die Vernetzung heterogener Elemente in einem Mediendispositiv deckt sich in Foucaults Dispositivbegriff mit der Verbindung von Sichtbarkeiten und Sagbarkeiten. Die „Schichten oder historischen Formationen“, die Foucault mit seiner Diskursarchäologie freizulegen vermag, bestehen stets aus einer Art des Sagens und einer Weise des Sehens, aus „Diskursivitäten und Evidenzen“ (Deleuze 1992: 69ff). Vor diesem Hintergrund entwickelt die Archäologie des Wissens den methodologischen Rahmen, die Elemente dieser Schichtungen freizulegen. Den diskursiven Formationen der Aussagen stehen im Dispositiv die Sichtbarkeiten als nicht-diskursive Formationen gegenüber. Ein irreduzibles, stets wechselseitiges Zusammenspiel der diskursiven Ordnungen und der ,Maschinen der Sichtbarkeit“, wie Gilles Deleuze betont hat:

„Sowenig die Aussagen ablösbar sind von ihren Ordnungen, so wenig sind die Sichtbarkeiten von ihren Maschinen ablösbar. Nicht dass jede Maschine von optischem Charakter wäre; aber es handelt sich um eine Zusammenstellung von Organen und Funktionen, die etwas sehen lässt, die ans Licht bringt, zur Evidenz [...].“ (Deleuze 1992: 83)

Bereits die Archäologie des Wissens operiert mit Diskursen und Evidenzen, denn „Foucault war stets ebenso fasziniert von dem, was er sah, wie von dem, was er hörte oder las, und die Archäologie, so wie er sie begriff, stellt ein audiovisuelles Archiv dar [...]“ (Deleuze 1992: 72f). Darin verortet Foucault das Konstitutionsmoment jedes Wissens: das Sichtbare und das Sagbare bilden das Objekt einer Epistemologie - und nicht einer Phänomenologie (vgl. ebd.). Als „Gesetz dessen, was gesagt werden kann“, als „,das allgemeine System der Formation und der Transformation der Aussagen“

3 Als Medien-Dispositiv findet das Mobiltelefon (neben dem Fernsehen) in Giorgio Agambens Text „Was ist ein Dispositiv?“ (2008: 37) explizite Erwähnung. Allerdings verzichtet Agamben auf eine medienwissenschaftlich vertiefende Beschreibung dieses Mediendispositivs und ,beschränkt' sich auf dessen Prozesse der Subjektivierung undDesubjektivierung 
(Foucault 1973: 187f) bildet das diskursive Archiv ein Dispositiv des Wissens, das durch die Sichtbarkeiten und ihre Maschinen ergänzt wird. Sagbares und Sichtbares müssen dabei weder übereinstimmen oder korrespondieren, noch eine gemeinsame Form annehmen. Vielmehr besteht ein Gegensatz zwischen Sehen und Sprechen: „was man sieht, liegt nie in dem, was man sagt“ (Deleuze 1992: 92). Das audiovisuelle Archiv ist disjunktiv; jede historische Formation ist von einem zentralen archäologischen Riss durchzogen. Gleichwohl bleiben in dieser Disjunktion Aussagen und Sichtbarkeiten in Verbindung: zwischen dem Sichtbaren und seiner Bedingung gleiten die Aussagen hin und her; und zwischen die Aussage und ihre Bedingung schleichen sich die Sichtbarkeiten ein (vgl. ebd.: 93ff). Die „Prozeduren des Wahren“ (ebd.: 91) bestehen so aus Praktiken des Sehens und Praktiken des Sagens, aus maschinellen Prozessen der Sichtbarkeit und Aussageverfahren, die zusammen Wissen konstituieren. Medien werden damit nicht nur als „das technische und logische Gesetz dessen [...], was heute sagbar ist" (Ernst 2004: 239), beschreibbar: die diskursiven Ordnungen und die Regime der Sichtbarkeit in Mediendispositiven sind fester Teil wissenskonstituierender, politischer Wahrheitsprozeduren.

Die Politik medialer Dispositive operiert mittels dieser zugleich disjunktiven und verbindenden, audiovisuellen Prägung. Was Foucault als die historischen Schichten formenden Arten des Sagens und Weisen des Sehens aufzeigt, bestimmt Jacques Rancière als die Politik einer „Aufteilung des Sinnlichen“, eines „Systems sinnlicher Evidenzen“:

„Die Aufteilung des Sinnlichen legt sowohl ein Gemeinsames, das geteilt wird, fest als auch Teile, die exklusiv bleiben. [...] Die Unterteilung der Zeiten und Räume, des Sichtbaren und Unsichtbaren, der Rede und des Lärms geben zugleich den Ort und den Gegenstand der Politik als Form der Erfahrung vor. Die Politik bestimmt, was man sieht und was man darüber sagen kann, sie legt fest, wer fähig ist, etwas zu sehen und wer qualifiziert ist, etwas zu sagen, sie wirkt auf die Eigenschaften der Räume und die der Zeit innewohnenden Möglichkeiten aus." (Rancière 2006: 25ff)

Den dispositiven Ordnungen medialer Elemente liegt aus der Perspektive Rancières eine politische Ästhetik zu Grunde: sie organisieren und transportieren eine Aufteilung des Sinnlichen, eine Ordnung von Diskursen und Evidenzen, die gleichermaßen die Existenz eines Gemeinsamen wie dessen Unterteilung bestimmt. Rancière hat diese Politik der Diskurse und Evidenzen exemplarisch an der Kunst verdeutlicht, die weder auf Grund ihrer Botschaften noch durch ihre Darstellung sozialer Strukturen oder ethnischer und sexueller Identitäten politisch wird, sondern durch ,eine Veränderung der Beziehung zwischen den Formen des Sinnlichen und den Regimen der Bedeutungszuweisung“:

„Kunst ist in erster Linie dadurch politisch, dass sie ein raum-zeitliches Sensorium schafft, durch das bestimmte Weisen des Zusammen- oder Getrenntseins, des Innen- 
oder Außen-, Gegenüber- oder in-der-Mitte-Seins festgelegt werden. Kunst ist dadurch politisch, dass sie einen bestimmten Raum und eine bestimmte Zeit aufteilt, und dass die Gegenstände, mit denen sie diesen Raum bevölkert, und die Rhythmen, in die sie diese Zeit einteilt, eine spezifische Form der Erfahrung festlegen, die mit anderen Formen der Erfahrung übereinstimmt oder mit ihnen bricht." (Rancière 2006: 77)

Die Aufteilung des Sinnlichen, des Sag- und Sichtbaren in Mediendispositive hat damit insofern politischen Charakter, als ,sie zugleich die Existenz eines Gemeinsamen aufzeigt wie auch die Unterteilungen, durch die innerhalb dieses Gemeinsamen die jeweiligen Orte und Anteile bestimmt werden" (Rancière 2006: 25f). Rancières politische Ästhetik verweist damit auf die stets neu zu verhandelnde Irreduzibilität von Singularität und Alterität jeder Gemeinschaft, die sowohl aus Singularitäten, wie auch aus einer Repräsentation ihrer Allgemeinheit bestehen muss (vgl. Derrida 2000: 47). Das ,Politische“ medialer Dispositive liegt in ihrer Bestimmung des Gemeinsamen und der Anteile, in ihrer Strukturierung von Sag- und Sichtbarem; sie organisieren und bestimmen die „Erfahrung der (Mit-)Teilung der Gemeinschaft" (Nancy 1988: 105, zit. nach Bedorf 2010: 30) ${ }^{4}$.

Identifizieren und repräsentieren Medien das politische Prinzip des Gemeinschaftlichen ${ }^{5}$, kommt ihren dispositiven Anordnungen weiteres politisches Gewicht zu. Als Aufteilung des Sinnlichen, d.h. gegenseitigen Durchdringung von Sag- und Sichtbarkeiten, sind Mediendispositive jenen von Foucault beschriebenen Machtsystemen zu zurechnen, die Wahrheitsregime erzeugen und stützen:

„Als ,Wahrheit“ ist ein Ensemble an geregelten Prozeduren zu verstehen, das die Erzeugung, die Gesetzmässigkeit, die Verteilung, die Zirkulation und das Funktionieren von Äusserungen bestimmt. Die ,Wahrheit' ist zirkulär mit Machtsystemen verbunden, die sie hervorbringen und stützen, sowie mit Machtwirkungen, die sie induziert und welche sie fortführen - Wahrheits-,regime‘ " (Foucault 1999: 29)

4 Schon deshalb gibt es kein einseitiges Abhängigkeitsverhältnis von Politik und Medien. Politik ist a priori auf die Medialität der Medien angewiesen: Medien identifizieren und repräsentieren ein Gemeinschaftliches, ja machen es überhaupt erst fest- und vorstellbar. Als „gemeinschaftliches Dazwischen“ (Tholen 2002: 179ff) schafft Medialität jene notwendige Unterbrechung von Kommunikation, die eine (Mit-)Teilung zwischen Repräsentation und Singularität ermöglicht.

5 Auch die Cultural Studies haben auf diese ,erste grosse kulturelle Funktionen moderner Medien“ hingewiesen: „This is the first of the great cultural functions of the modern media: the provision and the selective construction of social knowledge, of social imagery, through which we perceive the ,worlds', the ,lived realities ' of others, and imaginarily reconstruct their lives and ours into some intelligible ,world-of-the-whole“, some, lived totality““( (Hall 1984: 340f). 
Die Aussageverfahren und maschinellen Prozesse der Sichtbarkeit operieren in Mediendispositiven als mediale Politiken der Auf- und Unterteilung des Sinnlichen $^{6}$ und der Wahrheit. Medientechnologische Entwicklungen oder Anpassungen in der Mediennutzung sind deshalb gleichermaßen politisch zu werten. Das moderne, hochauflösende Fernsehen macht ungeahnte Details kontrastreich und in fast surreal wirkender Schärfe sichtbar ${ }^{7}$ und Webplattformen wie Facebook oder Twitter organisieren die laufende Diskursivierung, Speicherung und Verbreitung tagebuchartiger, scheinbar belangloser Details aus dem Leben ihrer Nutzer.

\section{Die ,Polizei‘ medialer Dispositive}

Rancières ,Politik des Ästhetischen“, in deren Zentrum weniger die Ausübung von Macht als vielmehr die sinnliche Aufteilung eines spezifischen Raumes der gemeinsamen Angelegenheiten steht, ist in diesem Sinne zu erweitern. Denn für Mediendispositive ist gerade die strategische, machtkonstituierte wie -konstituierende Ausrichtung heterogener, medialer Elemente charakteristisch. Dispositive sind stets in ein „Spiel der Macht“ eingeschrieben und an „Grenzen des Wissens gebunden, die daraus hervorgehen, es gleichwohl aber auch bedingen“ (Foucault 1978b: 123). Die Kombinationen und Variationen von Sicht- und Sagbarkeiten erfahren nach Deleuze ihre entscheidende Ergänzung durch Kräftelinien, die eine ordnende Funktion einnehmen:

„Drittens schließt ein Dispositiv Kräftelinien mit ein. Man könnte sagen, dass sie in den vorangehenden Linien [der Aussagen und der Sichtbarkeiten $(\mathrm{SaS})$ ] von einem singulären Punkt zum anderen verlaufen; sie ,richten ' die vorangehenden Kurven gewissermaßen ,neu aus', sie ziehen Tangenten und entwickeln Verlaufskurven von einer Linie zur anderen, bewerkstelligen das Kommen-und-Gehen vom Sehen zum Sprechen und anders herum, wobei sie wie Pfeile agieren, die unablässig die Worte und Dinge durchkreuzen und nicht aufhören, um diese den Kampf zu führen.“ (Deleuze 2005: 154)

6 Bezeichnenderweise hat Rancière selbst die Aufteilung des Sinnlichen an weiteren Medienbeispielen verdeutlicht: so an der Demokratie des Romans, d.h. in der „gleichgültige[n] Demokratie der Schrift, so wie sie vom Roman im Verhältnis zu seinem Publikum symbolisiert wird“ oder in der abstrakten Malerei, die nicht die Entdeckung der zweidimensionalen Oberfläche als ein ureigenes Medium bestimmt, sondern als Oberfläche selbst eine Aufteilung des Sinnlichen darstellt (vgl. Rancière 2006: 29ff).

7 Markus Stauffs und Judith Keilbachs Ansatz in diesem Band, ,Veränderung ‘ und ,Transformation“ als fundamentale Merkmale des Mediums Fernsehen aufzufassen, erscheint vor diesem Hintergrund besonders vielversprechend. 
Jede Ordnung der Diskurse und Evidenzen hat strategischen Charakter und Mediendispositive richten ihre heterogenen Elemente stets mit spezifischen Zielen aus. Die Dispositive Foucaults und das apparative „Dispositiv Kino“ Jean-Louis Baudrys verbindet diese strategische Komponente: auch das Kino-Dispositiv ordnet - im Interesse des Realitätseindrucks für den Zuschauer - Apparate, Raum, Leinwand, Sitzordnung und Licht auf bestimmte Weise an (vgl. Paech 1997: 403) ${ }^{8}$. ,Politisch' sind die strategischen Mediendispositive deshalb nicht in erster Linie als Politik, sondern vielmehr als ,Polizei '. Mit Foucault lässt sich die politische Funktion medialer Dispositive als Ordnungsmacht beschreiben, deren Wirkung auf die gemeinschaftlichen Tätigkeiten der Individuen zielt:

„Die Rolle der Polizei als Form rationalen Einwirkens der politischen Macht auf Menschen besteht darin, diesen eine Art kleines Extra-Leben zu verschaffen, und indem sie das tut, gewährt sie dem Staat eine kleine Extra-Stärke. Dies geschieht mittels Kontrolle des ,Verkehrs', das heißt der gemeinschaftlichen Tätigkeiten der Individuen [...].“ (Foucault 1994: 87)

Die Aufteilung des Sinnlichen, das ,allgemein inbegriffene Gesetz, das die Formen des Teilhabens bestimmt, indem es zuerst die Wahrnehmungsweisen festlegt, in die sie sich einschreiben“ (Rancière 2008: 31) ist als solche Polizei und nicht als Politik zu verstehen. Erst ein Aufbrechen der Ordnungen der Sag- und Sichtbarkeit ist für Rancière genuin politisch und verdient den Namen einer Politik:

„Ich schlage nun vor, den Namen der Politik auf genau die bestimmte Tätigkeit [...] zu beschränken: diejenige, die die sinnliche Gestaltung zerbricht, wo die Teile und die Anteile oder ihre Abwesenheit sich durch eine Annahme definieren, die darin per definitionem keinen Platz hat: die eines Anteils der Anteillosen." (Rancière 2002: 41)

Es sind die Politik und die Polizei der Mediendispositive, die bei Rancière ,das Politische‘ bilden. Spätestens seit den 1980er-Jahren und den Arbeiten von Jean-Luc Nancy und Philippe Lacoue-Labarthe konstatiert die politische Philosophie jene grundlegende Differenz zwischen Politik (franz. la politique) und einem Politischen (franz. le politique). Zentrale Motivation dieser Differenzierung ist die Bestimmung des essentiell Politischen als philosophischem Gegenstand, die sich von einer politischen Theorie abgrenzt, die immer nur nach den Organisations- und Legitimationsformen bereits institutionalisierter Politik fragen kann (vgl. ausführlich Bedorf 2010). For-

8 So gelingt es Joachim Paech auch, das Dispositiv als „Theorie medialer Topik“ zu beschreiben: „Das Dispositiv ist die (topische) Ordnung, in der (z. B. audiovisuelle) Diskurse ihren Effekt (z. B. den Realitätseindruck) erzielen“ (Paech 1997: 410). 
men institutionalisierter Politik - vom Staat über das Parlament bis hin zur Demokratie - werden angesichts dieser politischen Differenz ebenfalls als eigene Mikrodispositive oder Elemente in Makrodispositiven denkbar, die Diskurse des Politischen auf je spezifische Weise ordnen und transformieren. Rancière verortet diese Differenz zwischen Politik und Polizei; das ,Politische' besteht gerade im Aufeinandertreffen von „Regierung im weiten, fast Foucault'schen Sinne des Begriffs der Regierungstechnologien (la police) und [...] Emanzipation (la politique)“ (Marchart 2010: 180).

Politik im Sinne einer „Intervention in das Sagbare und Sichtbare“, als „Demonstration des Dissens“ (Rancière 2008: 32f) wird durch die Kräftelinien der Mediendispositive also gerade verunmöglicht. Niemals bleiben Medien bei einer blossen Repräsentation von Gemeinschaft: vielmehr ordnen und inventarisieren sie und entziehen zugleich ihre dispositive Struktur und strategische Ausrichtung jeder politischen Intervention oder Verhandlung. Die mediale Ordnung, Inventarisierung, Kartographierung oder Normierung des (imaginierten) Gemeinschaftlichen wird gemeinschaftlich weder geteilt noch mitgeteilt. Als Dispositive gedacht, verdeutlicht sich die heterogene Komplexität medialer Diskurse, Institutionen, Einrichtungen, Entscheidungen und Gesetze zur politischen Ordnungsmacht einer medialen ,Polizei“.

In dieser machtdurchzogenen Verunmöglichung einer Intervention findet sich schließlich auch die von Deleuze beschriebene vierte DispositivDimension, nämlich der „Individuierungsprozess“ der ,Subjektivierungslinien“ (Deleuze 2005: 155f). Die „Maschinen der Sichtbarkeit“, wie sie Foucault anhand der Maschinen Roussels, aber auch anhand des panoptischen Gefängnisses und des klinischen Blicks beschrieben hat, sind Dispositive von Aussagen und Sichtbarkeiten, die Wissen und Subjektivitäten formieren. Die Bedingung der Sichtbarkeit in Mediendispositiven ist dementsprechend keine subjektive Sichtweise; das Subjekt ist vielmehr ,,seinerseits eine Stelle innerhalb der Sichtbarkeit, eine abgeleitete Funktion der Sichtbarkeit [...]“ (Deleuze 1992: 82). Diskursiv finden sich die Subjektivierungsprozesse in der strategischen Interdiskursivität des Dispositivs: ihr Geflecht aus Elementar- und Spezialdiskursen generiert laufend ,kompatible Subjektivität und reintegrierende Diskurselemente“ einer „Kollektivsymbolik“ (Link 2001: 78). Die mediale ,Polizei` basiert damit auf jenem produktiven $\mathrm{Zu}$ sammenspiel von Macht und Wissen, das Foucault ins Zentrum seines Dispositivbegriffs gestellt hat. Mediendispositive repräsentieren, ordnen und subjektivieren: als Wahrheitsregime diktieren sie Handlungs- und Wahrnehmungsweisen im Feld des Politischen, das sie zugleich freisetzen und einschränken. Sie operieren mit spezifischen „Subjektivierungslinien“, mit einem „Individuierungsprozess“ des Selbst, ,der sich auf Gruppen oder Personen bezieht und sich den etablierten Kräfteverhältnissen sowie den konstituierten Wissensarten entzieht“" (Deleuze 2005: 155f). Der abendliche Fernsehzuschauer wird als ,zappeur“ (de-)subjektiviert (vgl. Agamben 2008: 37), die Internetbesucherin als Kommentatorin ihres Privatlebens und der 
Computerspieler als Operator zwar narrativ gerahmter, aber maschinell vorgegebener Steuerbefehle. Genau deshalb werden Mediendispositive auch Teil gouvernementaler Machtbeziehungen; sie transportieren und regulieren gleichermaßen Regierungspraktiken und Selbsttechnologien. Im Zeittaler der Gouvernementalität werden Medien zu ,handhabbaren Gegenständen mit ,Potenzialen“ und ,Gefahren' [...], deren rationale Handhabung wiederum weitere Gegenstände - Praktiken, Familienverhältnisse, Subjektivitäten, Bevölkerung - reg(ul)ierenden Zugriffen zugänglich macht" (Stauff 2005: 108). Die Gouvernementalität der Medien gründet in ihrer disponierenden ,Polizei‘: Mediendispositive zählen zu jenen „Institutionen, [...] Verfahren, Analysen und Reflexionen, den Berechnungen und den Taktiken“, deren komplexe Machtform ,als Hauptzielscheibe die Bevölkerung, als Hauptwissensform die politische Ökonomie und als wesentliches technisches Instrument die Sicherheitsdispositive hat" (Foucault 2000: 64f). Zu Recht hat Christoph Engemann deshalb Gouvernementalität zu einer „Gouvernemedialität" weitergedacht:

„Über Medien geben sich Individuen und der Staat jenes Wissen über sich selbst und andere, das Gegenstand der modernen Regierungen ist. Regierungswissen ist ohne Medien nicht denkbar und moderne Regierungen zeichnen sich dadurch aus, dass sie die Medien selbst zum Gegenstand ihrer Politik machen.“ (Engemann 2011)

Die selbst- und regierungstechnologische Funktion einer medialen Ordnungsmacht verdeutlicht sich in (inter-)medialen Mikrodispositiven genauso wie den medial geprägten Makrodispositiven. Das moderne Mobiltelefon ,diktiert“ via Textnachrichten und ständiger Erreichbarkeit Kommunikationsrhythmen, verortet mittels Satellitennavigation auf interaktiven Karten, ,verbildlicht" dank eingebauter Kamera den Alltag und verpflichtet zugleich durch Netzanbindung, anwendungsfreundliche Applikationen und stete Verbindung zu ,sozialen` Online-Portalen zur ständigen Mitteilung. Gleichsam finden sich die gouvernementalen Machttechniken auf Ebene der Makrodispositive wieder, wie Johanna Dorer anhand des historischen Übergangs von einem „Informationsdispositiv“ hin zu einem „Kommunikationsdispositiv“ gezeigt hat: in der zweiten Hälfte des 20. Jahrhunderts verdrängen die technische Entwicklung der Massenmedien, das Aufkommen von Public Relations- und Werbediskursen und ein omnipräsenter, öffentlicher ,Geständniszwang' das von Zensur und Repression geprägte Informationsdispositiv und formen ein Kommunikationsdispositiv der ,ständigen mehrdimensionalen Rede“ und des „unablässigen Sprechens in der Medienöffentlichkeit“ (Dorer 1999: 302; ausführlich Dorer/Marschik 1993). Die umfangreichen Kommunikations- und Werbebemühungen politischer oder wirtschaftlicher PR sind vor diesem Hintergrund genauso als Interdiskurs eines medial geprägten Dispositivs zu sehen, wie die aktuell vieldiskutierten ,partizipativen“ Publikationsmöglichkeiten ,sozialer Medien` im ,interaktiven“ Web 2.0 . 


\section{Mediale Interventionen des Politischen}

Zeigt sich die Politik medialer Dispositive in der ordnenden, subjektivierenden, selbst- und regierungstechnologisch geprägten Aufteilung der Sag- und Sichtbarkeiten, so ist diese Macht der Medien wesentlich einer eingeschränkten Wahrnehmbarkeit medialer Dispositive geschuldet. Diese werden scheinbar erst in dem Moment reflexiv, ,in dem das Dispositiv problematisch wird, nicht mehr bruchlos funktioniert oder misslingt" (Paech 1997: 410) ${ }^{9}$. Auch Foucaults Dispositive der Sexualität und der Strafjustiz bleiben unbemerkt; ihre Aussagen verborgen, solange nicht ihre Bedingungen zu Tage gefördert werden; ihre Sichtbarkeiten unsichtbar, wenn deren Untersuchung bei den Objekten, den Dingen und den Sinnesqualitäten stehen bleibt (vgl. Deleuze 1992: 77ff).

Die eingeschränkte Wahrnehmungsmöglichkeit der Mediendispositive lässt sich in der Mediengeschichte durchgängig verorten: die dispositive Anordnung des Zaubertricks einer verschwindenden Frau auf der Theaterbühne verdeutlicht sich nach Joachim Paech erst bei dessen Verfilmung, in der ein Zauberkasten (in dem eine Frau verschwand) selbst verschwindet und sich in Filmkamera und -projektor ,totalisiert“ (Paech 1991: 773ff). Gleichsam befinden sich modernere „Medientechnologien in Auflösung“ (Stauff 2001), wenn trotz der diskursiven Evidenz der Medien als stabile Objekte eine stetige Streuung und Verflüchtigung derselben stattfindet und wir einer ,ununterbrochenen Umformung des medialen Systems und einer fortlaufenden Erneuerung seiner Komponenten“ gegenüberstehen (ebd.: 83).

Erst intermediale Transformationen und Repräsentationen ermöglichen überhaupt die Reflexion medialer Dispositive: das Fernsehprogramm auf dem Mobiltelefon durchbricht dessen Raumanordnung des Wohnzimmers, das Kunstwerk im Fernseh-Kulturmagazin ist den Techniken und Praktiken des Museums entrissen ${ }^{10}$ und das dreidimensionale Kino beginnt vielleicht gerade, dessen althergebrachte, zweidimensionale Leinwand $\mathrm{zu}$ demontieren $^{11}$. Anders als eine omnipräsente, mediale ,Polizei` scheint mediale ,Poli-

9 Bei Baudry ist die „Verbannung aller Spuren von (technisch-apparativer) Arbeit aus der Repräsentation und Rezeption“ (ebd.: 401) ebenfalls festes Charakteristikum des Dispositivs Kino.

10 Vgl. hierzu ausführlich den Beitrag von Nadja Elia-Borer in diesem Band.

11 Gegen ein ausschliessliches ,Reflexiv-Werden` medialer Dispositive und Technologien in Momenten von Störung und Dysfunktionalität spricht, dass Medien ihre technisch-apparative Dimension gelegentlich selbst ausstellen - beispielsweise, wenn sie sich ,durch diskursive Versprechen und technische Andeutungen als Vorstufe eine perfektionierten und somit endgültigen Mediums präsentieren“ (Stauff 2001: S. 84). Allerdings liegt nahe, dass in derartigen medialen ,Selbstthematisierungen' die Macht- und Subjekteffekte medialer Dispositive aussen vor bleiben. 
tik' im Sinne , des Politischen“ nur an medialen Bruchstellen und in Momenten intermedialer Transformationen möglich. Aus Perspektive der politischen Differenz ist die Anordnung der heterogenen Elemente in medialen Dispositiven vorab als institutionalisierte Politik oder als ,Polizei' und nicht als genuin ,politisch“ interpretierbar. ,Politisch` werden Mediendispositive dagegen im Moment eines „kontingenten Ereignis der Störung“ (Krasmann 2010: 81), wenn „,die natürliche Ordnung der Herrschaft unterbrochen ist durch die Einrichtung eines Anteils der Anteillosen“ (Rancière 2002: 24). Mediale Bruchstellen, Dysfunktionalitäten und Wandlungsprozesse sind damit nicht nur Ausgangspunkt der Fragen nach medienspezifischen Materialitäten und nach Prozessen einer synthetischen, formalen, transformationalen, oder gar ontologischen Intermedialität (vgl. Schröter 1998): sie bilden zugleich jenes Moment, das die disponierende (An-)Ordnung medialer Dispositive verdeutlicht, indem diese aus Einzeldispositiven neue ,Konglomerate" bilden.

Medienumbrüche als die genuin politischen Momente der Intervention und eines Anteils der Anteillosen festzusetzen, wird Foucault jedoch nicht gerecht. So sehr intermediale Transformationen ehemals feste mediale Rahmen zur Verhandlung stellen mögen: sie bleiben stets von der strategischen Prävalenz eines Dispositivs geprägt. Jedes Dispositiv hat zu einem gegeben historischen Zeitpunkt auf einen Notstand reagiert, hierin liegt seine strategische Natur. Dispositive zeichnen sich eben nicht nur durch eine Struktur und Vernetzung heterogener Elemente aus, sondern auch durch einen bestimmten Typ von Genese (vgl. Foucault 1978b: 120f). Die Prozesse der „funktionellen Überdeterminierung“ und der „strategischen Wiederauffüllung“ dieser Genese sind einerseits politisch, geht es doch um eine Readjustierung der heterogenen Elemente im Dispositiv. So mag beispielsweise das Internet in politischen Revolutionen denjenigen eine Stimme geben, die in den staatlich kontrollierten Massenmedien Fernsehen, Radio und Presse nicht zu Wort kommen. Andererseits zielen mediale Transformationen stets darauf, Widersprüche und Gegensätze wieder in Einklang zu bringen. Die Genese neuer Ordnungen der Sag- und Sichtbarkeit basiert auf der Harmonisierung gewollter und ungewollter Wirkungen - und ist damit immer von der bestehenden strategischen Zielsetzung eines Dispositivs geprägt. Politische Intervention bleibt selbst stets diskursiv und ,evident ${ }^{`}$ konstruiert und wird auch in ihrem politischsten Moment immer noch von einer strategisch ausgerichteten Polizei organisiert. Prozesse medialer ,Selbstthematisierung ", wie sie beispielsweise in Fernsehserien, Kinofilmen oder Computerspielen durch die Ausstellung der jeweils eigenen medialen Möglichkeiten zu beobachten sind (z.B. mittels der Thematisierung des Fernsehgeräts in einer Fernsehserie oder der narrativen Rahmung von Steuerungsoptionen in Computerspielen), sind deshalb gleichsam als strategisch-disponierende Effekte der Mediendispositive aufzufassen.

In den sich historisch wie aktuell transformierenden Mediendispositiven stehen Politik und ,Polizei` deshalb in einer ständigen, irreduziblen Bezie- 
hung: als ,Polizei“ repräsentieren sie Gemeinschaft, ordnen, normieren und selegieren die Aufteilungen der Diskurse und Evidenzen und subjektivieren durch Selbst- und Regierungstechnologien; als Politik müssen sie in die eigenen audiovisuellen Archive eingreifen und schaffen durch Umbrüche sowohl Dissens als auch Raum für Interventionen, der allerdings stets strategisch besetzt bleibt. Jede Kritik der medialen ,Polizei ‘ und jede Dekonstruktion medialer Politik bewegt sich auch deshalb zwangsläufig in jenem ,,privilegierten Gebiet" der Diskursarchäologie:

„Die Analyse des Archivs umfasst also ein privilegiertes Gebiet: gleichzeitig uns nahe, aber von unserer Aktualität verschieden, ist es die Randung der Zeit, die unsere Gegenwart umgibt, zu ihr einen Überhang bildet und sie anzeigt in ihrer Alterität; es ist das, was uns außerhalb von uns begrenzt. Die Beschreibung des Archivs entfaltet ihre Möglichkeiten (und die Beherrschung ihrer Möglichkeiten) ausgehend von Diskursen, die gerade aufgehört haben, die unsrigen zu sein [...].“ (Foucault 1973: S. 189)

Im Feld der Medien ist der Ausgangspunkt jeder Politik in diesem Sinne ein medienwissenschaftlicher. Einerseits, weil , das Politische' unbedingt auf eine Medialität als gemeinschaftliches Dazwischen angewiesen ist und andererseits, weil erst eine Dekonstruktion medialer wie medial geprägter Dispositive deren Politiken freizulegen vermag. Erst eine „Profanierung der Dispositive“ - das heisst das Verfahren, ,mittels dessen das, was in ihnen eingefangen und abgesondert wurde, dem allgemeinen Gebrauch zurückgegeben wird [...]" (Agamben 2008: 41) erlaubt politische Interventionen, die diesen Namen verdienen.

\section{LITERATUR}

Agamben, Giorgio, Was ist ein Dispositiv?, Zürich: Diaphanes 2008.

Baudry, Jean-Louis, „Das Dispositiv: Metapsychologische Betrachtungen des Realitätseindrucks“, in: Lorenz Engell/Oliver Fahle/Britta Neitzel/Claus Pias/Joseph Vogl (Hg.), Kursbuch Medienkultur. Die massgeblichen Theorien von Brecht bis Baudrillard. Stuttgart: Deutsche Verlags-Anstalt DVA 1999, S. 381-404.

Bedorf, Thomas, „Das Politische und die Politik - Konturen einer Differenz", in: ders./Kurt Röttgers (Hg.), Das Politische und die Politik, Frankfurt a.M.: Suhrkamp 2010, S. 13-37.

Crouch, Colin, Postdemokratie, Frankfurt a.M.: Suhrkamp 2008.

Deleuze, Gilles, Foucault, Frankfurt a.M.: Suhrkamp 1987.

Deleuze, Gilles, Lust und Begehren, Berlin: Merve 1996. 
Deleuze, Gilles, „Was ist ein Dispositiv?“, in: François Ewald/Bernhard Waldenfels (Hg.), Spiele der Wahrheit. Michel Foucaults Denken, Frankfurt a.M.: Suhrkamp 2005, S. 153-162.

Derrida, Jacques, Politik der Freundschaft, Frankfurt a.M.: Suhrkamp 2002.

Dorer, Johanna/Marschik, Matthias, Kommunikation und Macht. Public Relations - eine Annäherung, Wien: Verlag Turia \& Kant 1993.

Dorer, Johanna, „Das Internet und die Genealogie des Kommunikationsdispositivs: Ein medientheoretischer Ansatz nach Foucault", in: Andreas Hepp/Rainer Winter (Hg.): Kultur - Medien - Macht. Cultural Studies und Medienanalyse, Opladen und Wiesbaden: Westdeutscher Verlag 1999, S. 295-305.

Dörner, Andreas, Politainment. Politik in der medialen Erlebnisgesellschaft, Frankfurt a.M.: Suhrkamp 2005.

Engemann, Christoph, „Write me down, make me real - zur Gouvernemedialität digitaler Identität“ (Manuskript), erscheint in: Josef Wehner/Jan Passoth (Hg.): Web 3.0: Zur Vermessung des Internets, Wiesbaden: VS Verlag für Sozialwissenschaften 2011.

Ernst, Wolfgang, „Das Gesetz des Sagbaren. Foucault und die Medien“, in:

Peter Gente (Hg.): Foucault und die Künste, Frankfurt a.M.: Suhrkamp 2004, S. 238-259.

Foucault, Michel, Archäologie des Wissens, Frankfurt a.M.: Suhrkamp 1973.

Foucault, Michel, Überwachen und Strafen. Die Geburt des Gefängnisses, Frankfurt a.M.: Suhrkamp 1977.

Foucault, Michel, Der Wille zum Wissen (Sexualität und Wahrheit, Bd. 1), Frankfurt a.M.: Suhrkamp 1987.

Foucault, Michel, Sicherheit, Territorium, Bevölkerung. Geschichte der Gouvernementalität I, Frankfurt a.M.: Suhrkamp 2004.

Foucault, Michel, „Recht der Souveränität/ Mechanismus der Disziplin“ [Vorlesung vom 14. Januar 1976], in: ders.: Dispositive der Macht. Über Sexualität, Wissen und Wahrheit, Berlin: Merve 1978 [= Foucault 1978a], S. 75-95.

Foucault, Michel, „Ein Spiel um die Psychoanalyse. Gespräch mit Angehörigen des Departement der Psychoanalyse der Universität Paris/Vincennes“, in: ders., Dispositive der Macht. Über Sexualität, Wissen und Wahrheit, Berlin: Merve 1978 [= Foucault 1978b], S. 117-175.

Foucault, Michel, „Die politische Funktion des Intellektuellen“, in: ders., Botschaften der Macht, Stuttgart: Deutsche Verlags-Anstalt DVA 1999, S. 22-29.

Foucault, Michel, „Omnes et singulatim. Zu einer Kritik der politischen Vernunft“, in : Joseph Vogl (Hg.), Gemeinschaften. Positionen zu einer Philosophie des Politischen, Frankfurt a.M.: Suhrkamp 1994, S. 65-93. 
Foucault, Michel, Die Gouvernementalität, in: Ulrich Bröckling/Susanne Krasmann/Thomas Lemke (Hg.), Gouvernementalität der Gegenwart. Studien zur Ökonomisierung des Sozialen, Frankfurt a.M.: Suhrkamp 2000 .

Foucault, Michel, „Die iranische Revolution breitet sich mittels Tonbandkassetten aus“, in: ders., Dits et Ecrits (Schriften), Bd. 3, Frankfurt a.M.: Suhrkamp 2003, S. 888-893.

Hall, Stuart: „Culture, The Media and The ,Ideological Effect““, in: James Curran/Michael Gurevitch/Janet Woollacott (Hg.): Mass Communication and Society, London: Edward Arnold, Open University Press 1984, S. 315-348.

Hans, Jan, „Das Medien-Dispositiv“, in: tiefenschärfe. Zeitschrift des Instituts für Medien und Kommunikation, WS 2001/2002 (2002), S. 22-28.

Krasmann, Susanne, „Jaques Rancière: Politik und Polizei im Unvernehmen“, in: Ulrich Bröckling/Robert Feustel (Hg.), Das Politische denken. Zeitgenössische Positionen, Bielefeld: transcript Verlag 2010, S. 77-98.

Link, Jürgen/Link-Heer, Ursula, „Diskurs/Interdiskurs und Literaturanalyse“, in: Zeitschrift für Literaturwissenschaft und Linguistik 77 (1990), S. 88-99.

Link, Jürgen, ,Aspekte der Normalisierung von Subjekten. Kollektivsymbolik, Kurvenlandschaften, Infografiken“, in: ders./Ute Gerhard/Ernst Schulte-Holtey (Hg.), Infografiken, Medien, Normalisierung. Zur Kartografie politisch-sozialer Landschaften, Heidelberg: Synchron Wissenschaftsverlag der Autoren 2001, S. 77-92.

Link, Jürgen, Versuch über den Normalismus: Wie Normalität produziert wird, Göttingen: Vandenhoeck \& Ruprecht 2006.

Marchart, Oliver, „Der Apparat und die Öffentlichkeit. Zur medialen Differenz von ,Politik“ und ,dem Politischen““, in: Daniel Gethmann/Markus Stauff (Hg.): Politiken der Medien, Zürich: Diaphenes 2005, S. 19-38.

Marchart, Oliver, Die politische Differenz: zum Denken des Politischen bei Nancy, Lefort, Badiou, Laclau und Agamben, Frankfurt a.M.: Suhrkamp 2010.

Meyer, Thomas, Mediokratie. Die Kolonisierung der Politik durch das Mediensystem, Frankfurt a.M.: Suhrkamp 2001.

Nancy, Jean-Luc, L'expérience de la liberteé, Paris: Galilée 1988.

Paech, Joachim, „Überlegungen zum Dispositiv als Theorie medialer Topik“, in: MEDIENwissenschaft 4 (1997), S. 400-420.

Paech, Joachim, „Eine Dame verschwindet. Zur dispositiven Struktur apparativen Erscheinens, in: Hans Ulrich Gumbrecht/Ludwig K. Pfeiffer (Hg.), Paradoxien, Dissonanzen, Zusammenbrüche. Situationen offener Epistemologie, Frankfurt a.M.: Suhrkamp 1991, S. 773-790. 
Rancière, Jacques, Das Unvernehmen. Politik und Philosophie, Frankfurt a.M.: Suhrkamp 2002.

Rancière, Jacques, Die Aufteilung des Sinnlichen. Die Politik der Kunst und ihre Paradoxien, Berlin: b_books 2006.

Rancière, Jacques, Zehn Thesen zur Politik, Zürich: Diaphanes 2008.

Schröter, Jens, „Intermedialität. Facetten und Probleme eines aktuellen medienwissenschaftlichen Begriffs“, in: montage/AV, Jg. 7, Nr. 2 (1998), S. 129-154.

Sieber, Samuel: „Politik und Medien in der Schleife der Iterationen. Medien in Krisen, Katastrophen und Revolten“, in: kultuRRevolution 60 (2011), S. 53-58.

Stauff, Markus, „Medientechnologien in Auflösung. Dispositive und diskursive Mechanismen von Fernsehen“, in: ders./Andreas Lösch/Dominik Schrage/Dierk Spreen (Hg.), Technologien als Diskurse. Konstruktionen von Wissen, Medien und Körpern, Heidelberg: Synchron Wissenschaftsverlag der Autoren 2001, S. 81-100.

Stauff, Markus, „Zur Gouvernementalität der Medien. Fernsehen als ,Problem“ und ,Instrument ““, in: Daniel Gethmann/Markus Stauff (Hg.): Politiken der Medien, Zürich: Diaphenes 2005, S. 89-110.

Thiele, Matthias, „Vom Mediendispositiv zum medialen Kombinat aus Dispositiven“, in: kultuRRevolution 55/56 (2009), S. 41-46.

Tholen, Georg Christoph, Die Zäsur der Medien, Frankfurt a.M.: Suhrkamp 2002.

Von Rohr, Matthieu, „Die Revolution, die keine war“, in: Spiegel Online, 31. Januar 2011, URL: http://www.spiegel.de/spiegel/0,1518,742430,00. html (letzter Zugriff am 3.6.2011). 